\title{
Perimeter-Minimizing Triple Bubbles in the Plane and the 2-Sphere
}

https://doi.org/10.1515/agms-2019-0004

Received September 14, 2018; accepted January 29, 2019

\begin{abstract}
We use continuous and discrete unification to prove that standard triple bubbles in $\mathbb{R}^{2}$ and $\mathbb{S}^{2}$ are the minimizers of perimeter, among all clusters (Definition 2.3) enclosing the same triple of areas.

Unification defines a unified measurement that allows all configurations, regardless of areas, to compete together. Continuous unification proves that if a unified minimizer were better than expected, it would have to have at least one interior bubble component. Discrete unification proves there can only be one interior bubble and that it must be connected. This leaves only the "daisy" configurations: one interior bubble surrounded by an even number of "petals." A more careful analysis also eliminates these, leaving only the standard triple bubbles as minimizers.
\end{abstract}

The result on the sphere is new; the result in the plane is due to Wichiramala [11]. The double bubble in the sphere was done by Masters [6].

Keywords: perimeter, minimization, bubble, cluster, triple, unification, reidentification, plane, 2-sphere

MSC: 49K10

\section{Introduction}

A unification proof works in the context of a family of minimization problems, each having its own constraints and its own proposed minimizer and target perimeter. Competitors are measured by the ratio of their perimeter divided by their target, based on the constraints that they satisfy. The goal is to prove that if there existed a competitor that did better than its target, then there would have to exist a modified competitor that beat its own target by a wider margin than the first, thus preventing the existence of a minimizer, in contradiction to Theorem 2.3 of Morgan [7].

Allowing constraints to change by absorbing them into the measurement scheme frees up a variety of modification strategies, divided into the two broad categories of continuous and discrete.

The strategy of continuous unification is to grow or shrink a component if its pressure is not well balanced relative to its area. Specifically, Proposition 4.4 states that in a unified minimizer, curvatures must all be proportional between competitor and proposed minimizer.

Discrete unification addresses the everpresent possibility that one of the bubbles in a cluster might prefer to divide its appointed area into two or more separate components. Discrete unification seeks to capitalize on the inherent perimeter cost of any extra subdivision of components, by reidentifying which components belong to which bubble in a competitor cluster. Specifically, we prove the $(36,64)$-Lemma (Corollary 3.3), which shows that if an interior component of a bubble (i.e., a component completely surrounded by other bubble components) has $64 \%$ or less of the total area of the bubble to which it belongs, then we should reidentify that component to now belong to one of its neighboring bubbles, thereby erasing some perimeter. We then adjust the proposed minimizer so that its areas match the newly apportioned areas of the competitor. The

^Corresponding Author: Gary R. Lawlor: Department of Mathematics, Brigham Young University, USA, E-mail: lawlor@mathematics.byu.edu 
$64 \%$ threshold marks the point where we are assured of the competitor saving more perimeter than the proposed minimizer might save, so that the competitor could not have been the unified minimizer. Corollary 3.3 also addresses boundary components (i.e., components sharing positive-length boundary with the ambient region outside the cluster); a boundary component having at most $36 \%$ of its bubble's area can also be reidentified advantageously. (These percentages depend on the number of potential neighbors, so they would weaken for a quadruple bubble analysis.)

These restrictions limit the possible topologies of competitors. They also enhance Paolini and Tamagnini's isoperimetric inequality for clusters (Lemma 3.1 of [8]), stated herein as Proposition 4.1. The idea is to think of each bubble component as being surrounded by its own membrane, which bumps up against the membranes of its surrounding components. The whole cluster is also surrounded by an additional membrane. This means every interface now consists of two membranes. We apply the regular isoperimetric inequality in $\mathbb{R}^{2}$ or $\mathbb{S}^{2}$ to the membrane around each component and add the results. Half of this sum is then a lower bound on the total perimeter of the competitor. In the sphere, by Corollary 2.17, whenever this lower bound is greater than 11.47 we know the competitor is not a minimizer.

The reason why the double membrane idea is strengthened by discrete unification is that if one component of a disconnected bubble could be very tiny compared to another component, the double membranes estimate could not take much (if any) advantage of our knowledge that the bubble is disconnected. But discrete unification prevents an area ratio larger than 16 to 9 among components of the same bubble. It turns out, then, that the resulting double-membrane perimeter bounds are only slightly weaker than what we would get if we knew the best case scenario, in which components would have equal areas. We use double membranes in the proofs of Propositions 4.6 and 4.9.

Continuous unification appears to have been first used by Rubinstein and Thomas [9] in the context of proving length minimization for networks. For multiple bubbles, it was introduced (under the name unifcation) in [5]. Discrete unification, which we introduce in the present article, has a precursor in a method called weak minimizers, employed by Foisy, et. al. [4], Wichiramala [11], and Tamagnini [10]. With the weak approach, competitor bubbles are allowed to have greater areas than their counterparts in the model cluster, although their target perimeter is not adjusted accordingly, as it is with the unification approach we outline here. Tamagnini goes a step further, reidentifying components as we do herein, but then having to scale the entire competitor so that the areas will all equal or exceed those of the model. The advantage in the present paper is that we can change the model along with the competitor. Since one has better control over the individual areas and perimeter of the model, the overhead cost will now be leaner.

An important part of the work involved in a unification proof lies in investigating the whole space of constraint sets, defining proposed minimizers (Definition 2.12), and ensuring that we can move smoothly within that space; see Section 2. A difficulty in this area occurs in the quadruple bubble problem. When a growing bubble attains area equal to that of another bubble $K$ in the cluster, sometimes it will not grow further and still be part of a proposed minimizer. Instead, the other bubble must take over the growth from there. This amounts to switching the identities of the two bubbles at the point when their areas become equal. The identity switch causes a discontinuity in the curvature - and thus in the rate of change of perimeter with respect to area - and thus weakens the benefits of continuous unification. But we will see that this problem does not happen with triple bubbles.

\section{The field of competitors and models}

Definition 2.1. Let $\mathbf{v}$ be a vertex of a regular tetrahedron and $W$ the tripod of rays extending along the three edges of the tetrahedron incident to $\mathbf{v}$. Given three positive radii $r_{1}, r_{2}, r_{3}$, find the points $\left\{\mathbf{c}_{i}\right\}$ on the respective edges of $W$ whose distances from $\mathbf{v}$ are $\left\{r_{i}\right\}$. By the law of cosines, the distance from $\mathbf{c}_{i}$ to $\mathbf{c}_{j}$ is $\sqrt{r_{i}^{2}+r_{j}^{2}-r_{i} r_{j}}$.

Let $P$ be the plane containing the points $\left\{\mathbf{c}_{i}\right\}$. Rotate each triangle $\Delta \mathbf{c}_{i} \mathbf{c}_{j} \mathbf{v}$ outward from the tripod and down into $P$, holding $\mathbf{c}_{1}, \mathbf{c}_{2}$ and $\mathbf{c}_{3}$ fixed each time. Let the resulting rotated images of $\mathbf{v}$ be called $\mathbf{c}_{i j}$. These will be junction points in the planar triple bubble that we now form. 

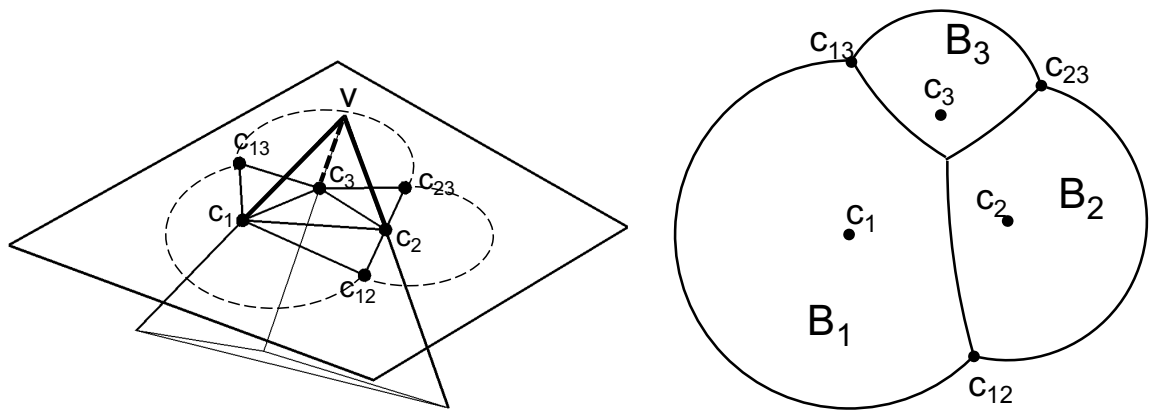

Figure 1

For $i \in\{1,2,3\}$, for each point $\mathbf{x} \in P$ define

$$
f_{i}(\mathbf{x})=\frac{\left\|\mathbf{x}-\mathbf{c}_{i}\right\|^{2}-r_{i}^{2}}{2 r_{i}}
$$

and also let $f_{0}$ be identically zero. For each $i \in\{0,1,2,3\}$ let $B_{i}$ be the generalized Voronoi cell defined as the set of points

$$
B_{i}=\left\{\mathbf{x} \in P: f_{i}(\mathbf{x})<f_{j}(\mathbf{x}) \text { for } i \neq j\right\} .
$$

Let the ordered triple $\left(B_{1}, B_{2}, B_{3}\right)$ define a standard triple bubble $T=T\left(r_{1}, r_{2}, r_{3}\right)$ in $P$, and call $B_{0}$ the exterior of the triple bubble. See Figure 1.

The defining radii of $T$ are $r_{i}$, the defining disks are the disks of radius $r_{i}$ centered at $\mathbf{c}_{i}$, and the defining centers are the centers of the defining disks.

This definition extends continuously to the limit as one or two radii go to zero, giving a standard double or single bubble; we include these also as (degenerate) triple bubbles for the sake of compactness.

Definition 2.2. Define the standard triple bubbles in the unit sphere $\mathbb{S}^{2}$ to be the images of standard planar triples under inverted stereographic projection $\sigma$. A triple bubble actually divides $\mathbb{S}^{2}$ into four components; by definition we will always choose one with largest area to be the exterior and the other three to be the "main bubbles." Defining disks are the images of defining disks in the plane, defining radii are geodesic radii on the sphere, and defining centers are centers (on the sphere) of the geodesic disks.

Definition 2.3. A cluster in the plane or sphere is a standard triple bubble or a competing figure consisting of an ordered triple of disjoint open sets (the three bubbles of the cluster), each not necessarily connected. The area triple of a cluster is the ordered triple of areas of these open sets, taking the sum if a bubble is disconnected. The boundary of a cluster is the topological boundary of the union of open sets, and the perimeter is the total length of this boundary, counting interfaces between bubbles just once.

Proposition 2.4. Given a standard triple bubble $T$ in $\mathbb{R}^{2}$ or $\mathbb{S}^{2}$, the (topological) boundary $\partial T$ is a union of generalized circular arcs separating the bubbles from each other and from the exterior. These arcs meet at 120degree angles and their signed (geodesic) curvatures add to 0 as you move around a triple junction point; thus, the curvature of an interface equals the difference of exterior curvatures of the respective bubbles, and the interface bows into the bubble of larger area. 
Proof. We first argue concerning standard triple bubbles in $\mathbb{R}^{2}$. The gradients of the functions $f_{i}$ and their differences are

or

$$
\begin{gathered}
\nabla f_{i}=\frac{1}{r_{i}}\left(\mathbf{x}-\mathbf{c}_{i}\right) \\
\nabla\left(f_{i}-f_{j}\right)=\left(\frac{1}{r_{i}}-\frac{1}{r_{j}}\right)\left(\mathbf{x}-\frac{r_{j} \mathbf{c}_{i}-r_{i} \mathbf{c}_{j}}{r_{j}-r_{i}}\right) \text { if } r_{i} \neq r_{j},
\end{gathered}
$$

$$
\nabla\left(f_{i}-f_{j}\right)=\frac{1}{r_{i}}\left(\mathbf{c}_{j}-\mathbf{c}_{i}\right) \text { if } r_{i}=r_{j} .
$$

These vector fields grow outward from the points $\mathbf{c}_{i}$ and (when $r_{i} \neq r_{j}$ ) from

$$
\mathbf{p}_{i j}=\mathbf{x}-\frac{r_{j} \mathbf{c}_{i}-r_{i} \mathbf{c}_{j}}{r_{j}-r_{i}},
$$

and they are perpendicular to the level sets of the respective functions, which are circles. At junction points where $f_{i}=f_{j}=0$, the magnitudes of $\nabla f_{i}$ and of $\nabla f_{j}$ are both equal to 1 , and by the way we constructed the points $\left\{\mathbf{c}_{i}\right\}$, the angle between gradients at the junction points is $2 \pi / 3$. This means that the difference $\nabla f_{i}-\nabla f_{j}$ also has magnitude 1 there. Its form shows that it has constant magnitude on any circle centered at $\mathbf{p}_{i j}$, so $\nabla\left(f_{i}-f_{j}\right)$ is the outward unit normal on the interface between bubbles $B_{i}$ and $B_{j}$.

Since $\nabla\left(f_{i}-f_{j}\right)$ has magnitude 1 on the respective bubble boundaries, we see from (2.1) that the curvature of the interface is $1 / r_{i}-1 / r_{j}$. Inspection of the position of the center of the interface circle in comparison with the other two shows that as we go around the vertex where the three circles meet, the signed curvatures sum to $1 / r_{j}+\left(1 / r_{i}-1 / r_{j}\right)-1 / r_{i}=0$, and the interface arc bows into the larger bubble. Or, if $r_{i}=r_{j}$ then $f_{i}-f_{j}$ is a linear function and the interface is a line segment, and the signed curvatures add to $1 / r_{i}-1 / r_{i}+0$, which is again zero.

Now in the sphere, the images under $\sigma$ of generalized circular arcs are circular arcs, and angles are preserved. For the curvature condition, we use the following argument.

Deleting one center point $\mathbf{c}_{i}$ in the plane and forming the Voronoi cells for the other two, we obtain a standard double bubble. Its image under $\sigma$ is a standard double bubble in $\mathbb{S}^{2}$, which, by [6], is perimeterminimizing and thus has first variation zero, meaning that its arcs satisfy the curvature condition wherever they meet. This argument covers the curvature condition at three of the four vertices in a triple bubble on $\mathbb{S}^{2}$. The fourth follows algebraically from the other three.

Finally, the result about bowing for clusters on the sphere is found in Lemma 2.5 below.

Lemma 2.5. On $\mathbb{S}^{2}$, let $B_{i}$ and $B_{j}$ be two bubbles, or one bubble and the exterior, of a standard triple bubble. If the area of $B_{i}$ is smaller than that of $B_{j}$ then the arc forming the interface between $B_{i}$ and $B_{j}$ bows outward from $B_{i}$ into $B_{j}$.

Proof. Begin with the configuration $B^{\prime}$ whose bubbles are the Voronoi cells $\left\{B_{i}^{\prime}\right\}$ of four equidistant points on $\mathbb{S}^{2}$, forming an equal-area quadruple bubble (i.e., standard triple bubble plus exterior). Choose two bubbles $B_{i}^{\prime}$ and $B_{j}^{\prime}$, and let $P$ be the plane containing the $\operatorname{arc} \alpha$ that separates $B_{i}^{\prime}$ from $B_{j}^{\prime}$.

Choose any point c outside $\mathbb{S}^{2}$ lying on the same side of $P$ as $B_{j}^{\prime}$, and let $C$ be the sphere centered at $\mathbf{c}$ that intersects $\mathbb{S}^{2}$ orthogonally. Let $F$ denote the spherical inversion across $C$.

For every point $\mathbf{p}$ in $B_{j}^{\prime}$, its reflection $R(\mathbf{p})$ across $P$ lies in $B_{i}^{\prime}$ and is further from $\mathbf{c}$ than the original point $\mathbf{p}$, so the Jacobian of $F$ is greater at $\mathbf{p}$ than at $R(\mathbf{p})$. This means that the area of $F\left(B_{j}^{\prime}\right)$ is greater than that $F\left(B_{i}^{\prime}\right)$.

Now extend the great circle arc $K$ between $B_{i}^{\prime}$ and $B_{j}^{\prime}$ to a full great circle, and make the same argument as before; the image of the hemisphere nearer to $\mathbf{c}$ will have greater area than the image of its counterpart, meaning that the circular arc $F(K)$ bows outward from $F\left(B_{i}^{\prime}\right)$ into $F\left(B_{j}^{\prime}\right)$.

Finally, given a nondegenerate triple bubble $U$ on $\mathbb{S}^{2}$, if there exists a spherical inversion that maps it to (a rotation or reflection of) $B^{\prime}$, then applying the above argument to the same inversion we obtain the desired result. To see that there is such an inversion, take the three spheres $S_{1}, S_{2}, S_{3}$ perpendicular to $\mathbb{S}^{2}$ whose intersections with $\mathbb{S}^{2}$ contain the exterior arcs of $U$. Find the point $\mathbf{q}$ of $S_{1} \cap S_{2} \cap S_{3}$ that lies outside 
$\mathbb{S}^{2}$, and find the sphere $D$ centered at q perpendicular to $\mathbb{S}^{2}$. Spherical inversion across $D$ maps each $S_{i}$ to a plane perpendicular to $\mathbb{S}^{2}$, so the image of the triple bubble is bounded by great circle arcs and, having four components, must be a rotation/reflection of $B^{\prime}$.

For a degenerate "triple" bubble enclosing only two nonzero areas plus the exterior, start with the Voronoi cells of three equidistant points on the equator, and make the same argument. For a degenerate single bubble, the result is trivial.

Proposition 2.6. Any equilibrium triple bubble $T$ having the same combinatorial type (six edges meeting in threes in the same way as the standard) is a standard triple bubble.

Proof. The hypothesis of equilibrium implies that $T$ is bounded by generalized arcs of circles meeting at 120 degrees (Theorem 2.3 of [7]). The exterior arcs have centers and radii, and this together with the angle criterion dictates the distances between the centers. So the outside shell (i.e., the boundary of the closure of the union of open sets that form the bubbles) matches the shell of a standard triple bubble $T_{2}$ with the given radii. Also determined are the directions of arcs starting at the junction points of the outer circular arcs and moving inward toward the middle of $T$, because of the 120-degree condition on arc junctions. But equilibrium also requires the zero sum of signed curvatures around each vertex (Theorem 4.1 of [3], Theorem 1 of [2]). Thus, the curvatures of the inward arcs are determined as well, and everything matches $T_{2}$.

We will need some facts about the growth of bubbles as one radius changes. We begin with the following lemma.

Lemma 2.7. Let $\mathbf{p}$ be a point on a line $L$ in $\mathbb{R}^{3}$ not coplanar with the $x$ axis. Rotate $L$ around the $x$-axis and for each rotation angle $\theta=\theta(t)$ let $\mathbf{p}_{\theta}$ and $L_{\theta}$ be the rotated images of $\mathbf{p}$ and $L$, and let $\mathbf{q}(t)$ be the point of intersection of $L_{\theta}$ with the $(x, y)$-plane. If the distance between $\mathbf{p}_{\theta}$ and $\mathbf{q}(t)$ increases at unit speed, then $\mathbf{q}(t)$ moves slower than unit speed in the $(x, y)$-plane.

Proof. Given a point of time at which we want to verify the result, call that point $t=0$ and parametrize the line $L$ as $\ell(t)=(a, b, 0)+t\left(v_{1}, v_{2}, v_{3}\right)$ where $\left(v_{1}, v_{2}, v_{3}\right)$ is a unit vector. Let $\theta(t)$ be the function defined by the requirement that the point

$$
\mathbf{q}(t)=\left(\begin{array}{ccc}
1 & 0 & 0 \\
0 & \cos \theta(t) & -\sin \theta(t) \\
0 & \sin \theta(t) & \cos \theta(t)
\end{array}\right)\left(\begin{array}{l}
a+t v_{1} \\
b+t v_{2} \\
0+t v_{3}
\end{array}\right)
$$

stay in the $(x, y)$-plane. Differentiating this by $t$ at $t=\theta=0$, we have

$$
\begin{aligned}
\mathbf{q}^{\prime}(t) & =\left(\begin{array}{ccc}
0 & 0 & 0 \\
0 & -\sin \theta(0) & -\cos \theta(0) \\
0 & \cos \theta(0) & -\sin \theta(0)
\end{array}\right)\left(\begin{array}{l}
a \\
b \\
0
\end{array}\right) \theta^{\prime}(0)+\left(\begin{array}{ccc}
1 & 0 & 0 \\
0 & \cos \theta(0) & -\sin \theta(0) \\
0 & \sin \theta(0) & \cos \theta(0)
\end{array}\right)\left(\begin{array}{l}
v_{1} \\
v_{2} \\
v_{3}
\end{array}\right) \\
& =\left(\begin{array}{ccc}
0 & 0 & 0 \\
0 & 0 & -1 \\
0 & 1 & 0
\end{array}\right)\left(\begin{array}{l}
a \\
b \\
0
\end{array}\right) \theta^{\prime}(0)+\left(\begin{array}{lll}
1 & 0 & 0 \\
0 & 1 & 0 \\
0 & 0 & 1
\end{array}\right)\left(\begin{array}{l}
v_{1} \\
v_{2} \\
v_{3}
\end{array}\right)=\left(\begin{array}{c}
v_{1} \\
v_{2} \\
v_{3}+b \theta^{\prime}(0)
\end{array}\right) .
\end{aligned}
$$

But since $\theta(t)$ is being chosen so that the intersection point remains in the $(x, y)$-plane, the last coordinate is zero, so the velocity vector of the intersection point is just $\left(v_{1}, v_{2}, 0\right)^{T}$, the projection of the unit vector $\mathbf{v}$ onto the plane. Since the line is not coplanar with the $x$-axis, $v_{3}$ cannot equal zero, so this projection has length strictly less than 1 .

Lemma 2.8. If we grow one radius of a standard triple bubble and hold the other two centers and radii fixed, the center of the growing bubble moves at a rate slower than the growth of the corresponding radius. 
Proof. Refer to the tripod $W$ in the definition of triple bubbles. Hold two points fixed where $W$ intersects the plane of a triple bubble, and rotate $W$ around the line through the fixed points. Apply Lemma 2.7 to the third edge of $W$, with $\mathbf{p}_{\theta}$ being the vertex of the tripod and $q(t)$ the third center.

Definition 2.9. For a figure that is changing in time, we say that the figure grows around itself if the figure at any given time is contained in the figure at any later time, and if there is strict growth in the sense that at least some boundary points at the previous time have become interior points at the later time.

Lemma 2.8 now gives the following.

Corollary 2.10. If we hold two centers and radii of a standard triple bubble fixed and grow the third radius, the third bubble grows around itself, as does its defining disk. The other two bubbles shrink in area, but the cluster as a whole also grows around itself; thus, the total area increases.

Proof. Consider first the question in the plane. Without loss of generality, let radius 3 be the one increasing. We prove that the Voronoi function (see Definition 2.1; $f_{3}$ now depends also on $t$ since the center $\mathbf{c}_{3}$ is moving) for the growing bubble has a negative (time) derivative at any fixed point in the plane. Once we know this, then since the other three Voronoi functions (including the constant function $f_{0}$ for the exterior) are fixed, this will cause the bubble to grow. Also, since $f_{0}$ is fixed, the shrinking of $f_{3}$ will cause the whole cluster to grow around itself.

The speed at which the boundary of the Voronoi cell moves outward equals the time rate of change of the Voronoi function divided by the magnitude of the (spatial) gradient of the function, so the outward movement will then be at positive speed all around the exterior boundary of bubble 3 .

Fix a point $\mathbf{x} \in \mathbb{R}^{2}$. Let $d_{i}(t)$ be the distance function $\left\|\mathbf{x}-\mathbf{c}_{i}(t)\right\|$ and $h_{i}(t)$ the function

$$
h_{i}(t)=f_{i}(\mathbf{x}, t)=\frac{d_{i}(t)^{2}-r_{i}(t)^{2}}{r_{i}(t)}
$$

at time $t$. Let $r_{i}(t)$ be growing at unit speed. Then

$$
h_{i}^{\prime}(t)=\frac{2 r_{i}(t) d_{i}(t) d_{i}^{\prime}(t)-d_{i}(t)^{2}-r_{i}(t)^{2}}{r_{i}(t)^{2}} .
$$

Since the point $\mathbf{c}_{i}$ moves slower than unit speed, the distance $d_{i}(t)$ from $\mathbf{x}$ to $\mathbf{c}_{i}(t)$ also changes slower than unit speed, so

$$
\begin{gathered}
d_{i}^{\prime}<1 \\
d_{i}^{2}\left(1-d_{i}^{\prime 2}\right)>0 \\
\left(r_{i}-d_{i} d_{i}^{\prime}\right)^{2}+d_{i}^{2}-d_{i}^{2} d_{i}^{\prime 2}>0 \\
r_{i}^{2}-2 r_{i} d_{i} d_{i}^{\prime}+d_{i}^{2}>0,
\end{gathered}
$$

implying that

$$
h_{i}^{\prime}(t)<0,
$$

as desired.

Shrinkage of the two bubbles with fixed radii and fixed centers follows because the closure of bubble 3 at any time is contained in the interior of bubble 3 at any later time. Thus, bubbles 1 and 2 lose some area to the expansion of bubble 3 .

Now on $\mathbb{S}^{2}$, given a standard triple bubble $T_{1}$, hold two radii and centers fixed and grow the third, obtaining a new standard triple bubble $T_{2}$. Stereographically project both to obtain $U_{1}$ and $U_{2}$, respectively, in the plane. We don't know a priori whether the third radius in of $U_{2}$ is larger or smaller than that of $U_{1}$, but if it were the latter, then by the above argument the third defining disk in $U_{2}$ would be contained in that of $U_{1}$. The same containment would hold upon projecting back to $\mathbb{S}^{2}$, but then the third radius would have shrunk, rather than growing, in $\mathbb{S}^{2}$. So the third radius in $U_{2}$ is greater than that of $U_{1}$, giving the above results about containment. The containment facts prove the desired results about area. 
Proposition 2.11. For every area triple there exists a unique standard triple bubble (up to rigid motions) that encloses those areas. The defining radii vary continuously with the areas.

Proof. Montesinos Amilibia [1] proved existence and uniqueness for clusters in $\mathbb{R}^{n}$. For existence and uniqueness on $\mathbb{S}^{2}$ we argue as follows.

Let $F$ be the map from ordered triples of geodesic radii to ordered triples of areas of standard clusters on $\mathbb{S}^{2}$. The domain of $F$ is $[0, \pi / 2]^{3}$, since a larger geodesic radius would cause the bubble to bow inward where it meets the exterior, which is prohibited by Lemma 2.5. The image consists of triple bubbles whose largest bubble has area less than or equal to that of the exterior. So within the region where (say) area $a_{1}$ is largest, we have the inequality $2 a_{1}+a_{2}+a_{3} \leq 4 \pi$, with similar inequalities where $a_{2}$ or $a_{3}$ are largest. This triple of inequalities in the nonnegative octant describes the range of $F$.

So each of the domain and range are six-sided solid polyhedra. Again by Lemma 2.5 the largest main area equals the exterior area if and only if their common boundary is a geodesic, if and only if that bubble's radius is $\pi / 2$. So $F$ maps face to corresponding face, edge to edge, and vertex to vertex. This means that $F$ has degree 1 and is surjective.

Now consider two different points $\mathbf{p}_{1}, \mathbf{p}_{2}$ in the domain of $F$. If all the coordinates of $\mathbf{p}_{1}-\mathbf{p}_{2}$ are either zero or have the same sign, then by Corollary 2.10, $F\left(\mathbf{p}_{1}\right) \neq F\left(\mathbf{p}_{2}\right)$. Now suppose two coordinates of $\mathbf{p}_{1}-\mathbf{p}_{2}$ have opposite sign; say radius 1 is larger in cluster 1 and radius 2 is larger in cluster 2 . Change the first cluster by shrinking radius 1 and growing radius 2 to now match the second cluster. By Corollary 2.10 this will shrink area 1 and grow area 2 in the first cluster. Now if radius 3 also needs to change, that will either increase both areas 1 and 2 or decrease both of them; either way, one of areas 1 and 2 will end up not matching the corresponding area in cluster 2. So $F$ is injective.

The continuity claim follows for $\mathbb{S}^{2}$ because $F$ is a bijection that is continuous in one direction, so it must be continuous in the other direction as well.

In $\mathbb{R}^{2}$ we define a map $\mathrm{G}$ from ordered triples of radii whose sum equals 1 , to ordered triples of areas of standard clusters in the plane. The domain is compact, the map is a bijection onto its image, and its inverse is continuous, so $\mathrm{G}$ is also continuous.

Definition 2.12. Given any cluster $C$ we define $M(C)$ to be the standard triple bubble having the same enclosed areas as $C$, and refer to $M(C)$ as the proposed minimizer related to $C$. We define the unified quotient measure $\mu$ by

$$
\mu(C)=\frac{P(C)}{P(M(C))},
$$

where $P$ denotes total perimeter of a cluster.

For closure we also include clusters enclosing only one or two positive areas, in which case $M(C)$ is a standard single or double bubble.

A unified minimizer is a minimizer of $\mu(C)$ over all competitors enclosing at least one positive area.

Proposition 2.13. Let $A(t)=\left(a_{1}(t), a_{2}(t), a_{3}(t)\right)$ be a smooth curve in $\mathbb{R}^{3+}$, and for each $t$ let $M(t)$ be the standard triple bubble in $\mathbb{R}^{2}$ or $\mathbb{S}^{2}$ enclosing that ordered triple of areas. Then the derivative of the perimeter of $M(t)$ is

$$
\frac{d}{d t} P(M(t))=\sum \kappa_{i}(t) a_{i}^{\prime}(t)
$$

where $\left\{\kappa_{i}(t)\right\}$ are the curvatures or geodesic curvatures along the exterior of the $i^{\text {th }}$ bubble of $M(t)$.

If we are working in $\mathbb{S}^{2}$ we temporarily waive the requirement that the exterior be largest, but we interpret curvature as being signed.

For a degenerate triple the proposition still holds, except that the derivative is undefined if we begin to inflate a zero-area bubble.

Proof. This follows because each $M(t)$ is in equilibrium and the radii vary continuously as a function of the ordered triple of areas enclosed, and is proved in Proposition 4.2 of [3]. 
Definition 2.14. The term reidentification refers to changing a competitor cluster by declaring one or more components in the cluster to now belong to a different bubble than before, and adjusting the model triple bubble so that its three bubble areas again match those of the competitor. One type we specifically call switch reidentification, which refers to trading the identities of two components.

The goal of reidentification is to guarantee that $M(C)$ does not save any more perimeter in the process than $C$ does. When we can do this, we know that the competitor $C$ was not a unified minimizer, as shown in the following.

Proposition 2.15. Suppose a (hypothetical) competitor $C$ has unified cost $\mu<1$. If there exists a modification of $C$ for which the perimeter goes down by $\alpha>0$ and if the corresponding modification of $M(C)$ required to match the areas again causes the perimeter of $M(C)$ either to go up, or to go down by an amount $\alpha^{\prime} \leq \alpha$, then $C$ is not a unified minimizer.

Proof. If the perimeter of $M(C)$ goes up, the result follows immediately. Otherwise, if

$$
\frac{P(C)}{P(M(C))}<1
$$

then

$$
\frac{P(C)-\alpha}{P(M(C))-\alpha^{\prime}} \leq \frac{P(C)-\alpha}{P(M(C))-\alpha}<\frac{P(C)}{P(M(C))}
$$

Proposition 2.16. If we change a standard triple bubble by bringing two unequal areas closer to their average, without changing their sum, then we increase the perimeter. As a result, if a competitor bubble (say bubble 1) has components of areas $a$ and $c$ and another (say bubble 2) has total area $b$, then if $b<c$ we can decrease the perimeter of the competitor by reidentification, while increasing that of the model.

Proof. Let $a_{i}<a_{j}$. By Proposition 2.4, bubble $i$ bows into bubble $j$, so $\kappa_{i}>\kappa_{j}$. Letting the derivative $a_{i}^{\prime}$ be positive and $a_{j}^{\prime}=-a_{i}^{\prime}$, by Proposition 2.13 the result follows. (If the original triple is degenerate, with one or more zero areas, the derivative is still positive except at the degenerate endpoint; this is sufficient for the proof.)

Now assume the hypotheses in the second paragraph of the corollary. Then clearly,

$$
|a+b-c|<a+c-b
$$

so that the area difference in the competitor's bubbles 1 and 2 decreases if we either switch the identities of the components having areas $b$ and $c$ (in the case $a+b \geq c$ ) or reidentify the component of area $a$ to now belong to bubble 2 (in the case $a+b<c$ ). Since the competitor now has total areas closer to their averages, when we make the corresponding changes in the model, the model perimeter goes up.

We now obtain the following important corollary.

Corollary 2.17. The maximum perimeter in $\mathbb{S}^{2}$ for a standard triple bubble is $6 \cos ^{-1}(-1 / 3)<11.47$. Therefore, any competitor on $\mathbb{S}^{2}$ with perimeter greater than 11.47 is not a minimizer.

Proof. By Proposition 2.16, the maximum perimeter of a triple bubble on $\mathbb{S}^{2}$ occurs for equal areas. Since that corollary applies equally well to the exterior bubble, it must also have area equal to the three main bubbles, so that each has an area of $\pi$. Then, the standard triple bubble bounded by geodesics is bounded by the six edges of a regular tetrahedron on the surface of $\mathbb{S}^{2}$, whose vertices are (for example) $1 / \sqrt{3}( \pm 1, \pm 1, \pm 1$ ), with an even number of minus signs. The angle (and thus arc length) between two of these is $\cos ^{-1}(-1 / 3)$, giving a total perimeter of $6 \cos ^{-1}(-1 / 3)$. 
Proposition 2.18. There exists a minimizer of $\mu$ among triple bubbles. It has these properties outlined in Theorem 1 of [2] and Theorem 2.1 of [11]:

1. All arcs have constant curvature.

2. Edges meet in threes at $120^{\circ}$-angles.

3. The sum of signed curvatures of the three arcs meeting at a node is zero.

4. There are pressures $p_{1}, \ldots, p_{m} \in \mathbb{R}$ such that every edge between $R_{i}$ and $R_{j}$ has curvature $p_{i}-p_{j}$ (bowing into the lower pressure region) where $p_{0}$ is set to be zero.

Proof. If $\mu$ is never less than 1 , the proposed minimizers of perimeter (all of which satisfy $\mu=1$ ) are the minimizers of $\mu$. So suppose that the infimum $\lambda$ of $\mu$ is less than 1 .

We work first in the plane. For each triple of areas, by Morgan's existence Theorem 2.3 of [7], there exists a minimizer of perimeter. Take a sequence of these minimizers with $\mu$ converging to $\lambda<1$. Scale each so that its total area equals 1 ; this does not change $\mu$.

A minimizer cannot be spread over a large area, because either the perimeter would be too large or the minimizer would be disconnected, and pieces could then be brought together to reduce perimeter. Thus, there exists a compact subset of the plane that contains each minimizer in the sequence. Thus we can take a subsubsequence that converges in the flat norm. Area and perimeter are continuous or lower semi-continuous in the flat norm, as is the perimeter of proposed minimizers of $\mu$, so the limit cluster has $\mu$ equal to $\lambda$, as desired.

The sphere is easier in the sense that it is already compact, but more difficult because we do not have a scaling function to normalize areas without changing $\mu$. The only case not covered by the same arguments as in the plane is the case where the sequence of area triples goes to $(0,0,0)$.

Again, minimizers with increasingly small perimeter must be contained in an increasingly small region; otherwise their perimeters would be too large or they would be disconnected. Scale the ambient spheres so that the clusters in the sequence now each have total area 1. For each scaled competitor, choose a plane tangent to the sphere at a point of the cluster, and flatten the cluster by orthogonal projection onto the tangent plane. The resulting change in perimeters and areas gets less and less, so we will have a limiting cluster, and $\mu$ will equal its infimum.

At this point there remains the possibility that a minimizer of $\mu$ is a single or double bubble. But since it is already known ([3] and [4]) that standard double bubbles (and of course single round bubbles) are the minimizers in the plane and sphere, and since we are assuming $\lambda<1$, it must be that $a_{i}>0$ for all $i$.

We now prove two identities that hold for $\mathbb{R}^{2}$ but not for $\mathbb{S}^{2}$.

Proposition 2.19. Let $T\left(r_{1}, r_{2}, r_{3}\right)$ denote a standard triple bubble of radii $r_{1}, r_{2}, r_{3}$ in $\mathbb{R}^{2}$. The total length of $\partial T\left(r_{1}, r_{2}, r_{3}\right)$ satisfies the Hutchings identity (see [3])

$$
P \equiv \operatorname{Len}\left(\partial T\left(r_{1}, r_{2}, r_{3}\right)\right)=2 \sum \frac{a_{i}}{r_{i}},
$$

where each $a_{i}$ is the area of $B_{i}$.

Proof. We give two proofs. One is by the paired divergence theorem. Apply the planar divergence theorem to each vector field $\nabla f_{i}$ on $B_{i}$, and add the results. Since the divergence of $\nabla f_{i}$ is the constant $2 / r_{i}$, and since the outward normals $\mathbf{n}_{i}$ and $\mathbf{n}_{j}$ are negations of each other along an interface,

$$
\begin{gathered}
2 \sum \frac{1}{r_{i}} a_{i}=\sum \int_{B_{i}} \operatorname{div} f_{i} d A=\sum \int_{\partial B_{i}} f_{i} \cdot \mathbf{n} d s=\sum_{i<j \in\{0,1,2,3\}} \int_{\partial B_{i} \cap \partial B_{j}}\left(f_{i} \cdot \mathbf{n}_{i}+f_{j} \cdot \mathbf{n}_{j}\right) d s \\
=\sum_{i<j} \int_{\partial B_{i} \cap \partial B_{j}}\left(f_{i}-f_{j}\right) \cdot \mathbf{n}_{i} d s=\sum \int_{\partial B_{i} \cap \partial B_{j}} 1 d s=\operatorname{Len}\left(\partial T\left(r_{1}, r_{2}, r_{3}\right)\right) .
\end{gathered}
$$

The second proof, due to Michael Hutchings, applies to any equilibrium bubble cluster. We simply dilate the configuration by a factor of $1+t$ for each $t$ near 0 , and note that on the one hand, the area $a_{i}(t)=(1+t)^{2} a_{i}$ 
while the perimeter $P(t)$ satisfies $P(t)=(1+t) P(0)$, so that the initial first derivatives are

$$
a_{i}^{\prime}(0)=2 a_{i} \text { and } P^{\prime}(0)=P(0) .
$$

On the other hand, by differential geometry the rate of change of perimeter equals the sum of exterior curvatures times the rates of change of the respective areas. So

$$
P^{\prime}(0)=\sum \frac{1}{r_{i}} a_{i}^{\prime}(0)
$$

Combining with the above we have

$$
P=P(0)=\sum \frac{2}{r_{i}} a_{i}
$$

The second proof generalizes to show that the $(n-1)$-dimensional surface area $S$ of an equilibrium cluster in $\mathbb{R}^{n}$, enclosing $n$-dimensional volumes $\left\{V_{i}\right\}$ and having mean curvatures $H_{i}$ where bubbles meet the exterior, satisfies

$$
S=\frac{n}{n-1} \sum H_{i} V_{i}
$$

We pause to note another beautiful identity to go along with the Hutchings identity.

Proposition 2.20 (The Susquehanna Identity). In the plane, the partial derivative of perimeter of standard triple bubbles by each radius is

$$
\frac{\partial P}{\partial r_{i}}=2 \frac{a_{i}}{r_{i}^{2}}
$$

The quantity $a_{i} /\left(\pi r_{i}^{2}\right)$ can be called the saturation of the bubble, being the fraction of the defining disk that it fills up. So another way to state this identity is that the partial derivative of perimeter by a radius is $2 \pi$ times the (current) saturation of the corresponding bubble.

Proof. By the chain rule,

$$
\frac{\partial P}{\partial r_{i}}=\sum_{j} \frac{\partial P}{\partial a_{j}} \frac{\partial a_{j}}{\partial r_{i}} .
$$

Then by differential geometry,

$$
\frac{\partial P}{\partial r_{i}}=\sum_{j} \frac{1}{r_{j}} \frac{\partial a_{j}}{\partial r_{i}} .
$$

Now differentiating the Hutchings identity by $r_{i}$, we get

$$
\frac{\partial P}{\partial r_{i}}=-2 \frac{a_{i}}{r_{i}^{2}}+\sum \frac{2}{r_{i}} \frac{\partial a_{i}}{\partial r_{i}} .
$$

Subtracting (2.4) from twice (2.3) we obtain the desired result.

\section{Discrete unification eliminates graphs with more than a few components}

We extend the utility of reidentification (Definition 2.14) as follows.

Lemma 3.1. Let $T_{1}$ and $T_{0}$ be model triple bubbles in $\mathbb{R}^{2}$ or $\mathbb{S}^{2}$, only one of whose bubble areas (say, the third bubble in each) differ. Let $a_{1}<a_{0}$ be the corresponding areas in the third bubble, and $P_{1 b}<P_{0 b}$ the total perimeters of $T_{1}$ and $T_{0}$. Let $P_{1 c}$ and $P_{0 c}$ be the perimeters of round disks having areas $a_{1}$ and $a_{0}$, respectively. Then

$$
P_{0 c}-P_{1 c}>P_{0 b}-P_{1 b} .
$$


Proof. Since each bubble in the model cluster is only a portion of the full disk from which it is cut, the radius of that disk is greater than the radius of the (full) disk having the same area. Thus, the exterior curvature $\kappa_{b}$ of that bubble (signed, in the $\mathbb{S}^{2}$ case) is less than the curvature $\kappa_{c}$ of the disk of the same area. But $\kappa_{b}$ is the derivative of total perimeter by the area of the third bubble, and $\kappa_{c}$ is the derivative of the disk's perimeter with respect to the enclosed area. Thus,

$$
P_{0 b}-P_{1 b}=\int_{a_{1}}^{a_{0}} \kappa_{b} d a<\int_{a_{1}}^{a_{0}} \kappa_{c} d a=P_{0 c}-P_{1 c} .
$$

Lemma 3.2. Let $\alpha_{1}, \alpha_{2}, \alpha_{3}>0$ with $\alpha_{1}>\alpha_{2}$ and $\alpha_{1}>\alpha_{3}$. For each $i \in\{1,2,3\}$, let $D_{i}$ be a disk of area $\alpha_{i}$ in $\mathbb{R}^{2}$ or $\mathbb{S}^{2}$, and let $P_{i}$ be the perimeter. Then

$$
\frac{\sqrt{\alpha_{2}}}{\sqrt{\alpha_{1}}-\sqrt{\alpha_{3}}}\left(P_{1}-P_{3}\right)<P_{2} \text {. }
$$

Proof. The proof for $\mathbb{R}^{2}$ follows immediately.

A disk on the sphere has a defining angle between the vectors $\mathbf{v}_{c}$ and $\mathbf{v}_{p}$, where $\mathbf{v}_{c}$ is the vector from the center of the sphere to the center of the disk and $\mathbf{v}_{p}$ is the vector from the sphere's center to any point of the boundary circle of the disk. If the defining angle of a disk is $\phi$ then the area is $2 \pi(1-\cos \phi)$ and the perimeter is $2 \pi \sin \phi$. Then the quotient of perimeter over the square root of area equals $\sqrt{2 \pi(1+\cos \phi)}$, which is a decreasing function. Since $\alpha_{1}>\alpha_{i}$ for $i \in\{2,3\}$,

$$
\frac{P_{1}}{\sqrt{\alpha_{1}}}<\frac{P_{2}}{\sqrt{\alpha_{2}}}
$$

and

$$
\frac{P_{1}}{\sqrt{\alpha_{1}}}<\frac{P_{3}}{\sqrt{\alpha_{3}}} .
$$

Then

$$
\frac{\sqrt{\alpha_{3}}}{\sqrt{\alpha_{1}}} P_{1}<P_{3}
$$

and

$$
\frac{\sqrt{\alpha_{1}}-\sqrt{\alpha_{3}}}{\sqrt{\alpha_{1}}} P_{1}<\frac{\sqrt{\alpha_{1}}-\sqrt{\alpha_{3}}}{\sqrt{\alpha_{2}}} P_{2}
$$

adding these, we get

$$
P_{1}<\frac{\sqrt{\alpha_{1}}-\sqrt{\alpha_{3}}}{\sqrt{\alpha_{2}}} P_{2}+P_{3}
$$

from which the result follows.

Corollary 3.3 (The $(36,64)$-Lemma). In a unified minimizer for the triple bubble in $\mathbb{R}^{2}$ or $\mathbb{S}^{2}$, no bubble can have an interior component with less than or equal to $64 \%$ of the area of the complete bubble, nor can any bubble have a boundary component with less than or equal to $36 \%$ of the complete bubble area. Consequently, a unified minimizer cannot have a bubble with two interior components, nor a bubble with one interior and one boundary component, nor a bubble with more than two boundary components.

In addition, for a minimizer in $\mathbb{S}^{2}$ the exterior cannot have a component with less than or equal to $36 \%$ of the exterior area.

Proof. Suppose a bubble (say, bubble 2) of total area $a$ has an interior component $K$ with precisely $64 \%$ of its area. Reidentify that component to belong to a different bubble (bubble $i$ ). Change the model by deflating bubble 2 from area $a$ to $\frac{9}{25} a$, temporarily holding the area of bubble $i$ fixed in the model. By Lemma 2.2 the resulting savings in perimeter for the model is less than the difference in perimeters of disks of area $a$ and $\frac{9}{25} a$, which, by Lemma 3.2 (with $\alpha_{1}=a, \alpha_{2}=\frac{16}{25} a$, and $\alpha_{3}=\frac{9}{25} a$ ) is less than half the perimeter of a disk 
of area $\frac{16}{25} a$; this, in turn, by the isoperimetric inequality, is less than half the perimeter of $K$. Now finish matching bubble areas between model and competitor by increasing the area of bubble $i$; this will increase model perimeter, which only enhances the desired inequality.

Now since $K$ is bounded by (components of) the other two bubbles, there is one of the bubbles whose common boundary with $K$ covers at least half the perimeter of $K$. Change the identity of $K$, then, to the bubble that borders it with more than half the perimeter of $K$. This allows us to erase that common boundary, saving more than the model saved from the overall reidentification.

Clearly any value of $\alpha_{2}$ smaller than $\frac{16}{25} a$, with $\alpha_{1}=a$, will also satisfy the same argument, thus eliminating components with less than $64 \%$ of a bubble's area.

Now by Proposition 2.15, if a competitor changes and saves more than the model saves, the original competitor could not have been the unified minimizer.

Next, suppose a bubble has an boundary component with at most $36 \%$ of its area. Set $\alpha_{1}=a, \alpha_{2}=\frac{9}{25} a$, and $\alpha_{3}=\frac{16}{25} a$ in Lemma 3.2. The result is that

$$
\frac{1}{3} P_{2}>\left(P_{0 b}-P_{1 b}\right) .
$$

Since the component is bounded now by three bubble components (of the other two bubbles and the exterior), we can only guarantee that one of them borders at least a third of the boundary of the component, which, by the above, is greater than $\left(P_{0 b}-P_{1 b}\right)$, and the argument follows along the same lines as before.

For bubbles in $\mathbb{S}^{2}$, this last argument works the same for the exterior bubble as for boundary components.

\section{Continuous unification eliminates graphs with lone leaves}

In this section we will apply the ideas from Paolini and Tamagnini, specifically the isoperimetric inequality for clusters, Lemma 3.1 of [8], already discussed in the introduction.

Proposition 4.1. The total perimeter of a cluster is at least half the sum of perimeters of disks whose areas equal the areas of the individual bubble components, plus half the perimeter of a disk whose area equals the total cluster area.

Proof. Apply the isoperimetric inequality to each component and then to the cluster as a whole. Think of each component as being enclosed by a membrane with a "thickness" of one half, with an additional halfthickness membrane enclosing the whole cluster. Then half the sum of membrane perimeters, which we can bound by the regular isoperimetric inequality, will amount to the total perimeter of the cluster.

Definition 4.2. A bubble triangle is a region bounded by three line segments or arcs of circles meeting at three vertices, such that the interior angle between any two of these segments or arcs is $120^{\circ}$.

Definition 4.3. A lone leaf is a component of a main bubble that touches only two other main bubble components, and is thus a bubble triangle.

We will see that if a minimizer did better than a standard triple, it could not have a lone leaf, because the leaf would have too much area.

Proposition 4.4. If $\lambda=\mu\left(C_{0}\right)<1$ is the minimum value of $\mu$ over all clusters with any triple of areas, then the geodesic curvatures of the pieces of $C_{0}$ are $\lambda$ times the geodesic curvatures of the corresponding pieces of $M\left(C_{0}\right)$, and the pressures (see Proposition 2.18) in $C_{0}$ are $\lambda$ times the corresponding pressures in $M\left(C_{0}\right)$.

Proof. Suppose that the statement does not hold. First, as has been noted, $C_{0}$ cannot be a single or double bubble, so all its areas are positive. 
Now some curvature $\kappa$ on $C_{0}$ is greater than (or, resp., less than) $\lambda$ times the curvature $\hat{\kappa}$ on the corresponding piece of $M\left(C_{0}\right)$; we define a family $C(t)$ by pushing inward (resp., outward) slightly on that arc of $C_{0}$. Then the function $\mu(C(t))$ initially decreases, contradicting the minimization property of $C_{0}$.

(Note: This inflation might hypothetically cause one bubble area on $\mathbb{S}^{2}$ to become larger than the exterior area, but this is not a problem because we simply switch the identity of a main bubble and the exterior, and the claim about perimeter is unaffected.)

The claim concerning pressures now follows from the definition of pressure.

We now need to establish connectivity of the exterior bubble. For planar clusters, when using unification this is trivial, because we can simply reidentify any bounded component belonging to the exterior as now belonging to one of the main bubbles, thus decreasing perimeter of the competitor while increasing the perimeter of the model. However, it is not as simple on $\mathbb{S}^{2}$, because such a reidentification might change which bubble is largest (and is thus the exterior) and might not increase the perimeter of the model.

Definition 4.5. Let iso $(x)=\sqrt{x(4 \pi-x)}$, the perimeter for a circle of area $x$ on the unit sphere $\mathbb{S}^{2}$.

Proposition 4.6. In a unified minimizer, the exterior is never disconnected.

Proof. As noted above, when using unification this fact is trivial in the plane.

For bubble clusters on the sphere, define the function

$$
f\left(x_{1}, x_{2}, y_{1}, y_{2}, y_{3}\right)=\frac{1}{2}\left(\operatorname{iso}\left(x_{1}\right)+\operatorname{iso}\left(x_{2}\right)+\operatorname{iso}\left(y_{1}\right)+\operatorname{iso}\left(y_{2}\right)+\operatorname{iso}\left(y_{3}\right)\right)
$$

where $x_{1}$ is the area of the smallest exterior component, $x_{2}$ is the sum of areas of remaining exterior components, and $y_{1}, y_{2}, y_{3}$ are the total areas of the three main bubbles. As in Proposition 4.1, $f$ gives a lower bound for the total perimeter.

Notice that $f$ has negative directional second derivatives in all directions. Thus, if we define a domain for $f$ that is bounded by hyperplanes, then the function will be minimized at some vertex of the domain.

By definition, the total exterior area is at least as large as each main bubble. By Corollary 3.3, if a piece of the exterior had less than $36 \%$ of the total exterior area we could improve by reidentification. By Proposition 2.16, if any main bubble had less area than the larger exterior component we could improve by reidentification. These three facts lead us to the domain $D$ in $\mathbb{R}^{5}$ lying on the hyperplane

$$
x_{1}+x_{2}+y_{1}+y_{2}+y_{3}=4 \pi
$$

and bounded by the inequalities

$$
\begin{gathered}
\frac{9}{16} x_{2} \leq x_{1} \leq x_{2}, \\
x_{2} \leq y_{1} \leq x_{1}+x_{2}, \\
x_{2} \leq y_{2} \leq x_{1}+x_{2}, \\
x_{2} \leq y_{3} \leq x_{1}+x_{2} .
\end{gathered}
$$

There are eight essentially different vertices of this polytope, which arise from selecting either the lefthand or the right-hand inequality on the first line and then, in the last three lines, choosing the left-hand inequality zero, one, two or three times. The resulting vertices (modulo permutations of the last three coordinates) are:

$$
\begin{aligned}
& \frac{\pi}{2}(1,1,2,2,2), \\
& \frac{\pi}{7}(4,4,4,8,8), \\
& \frac{\pi}{3}(2,2,2,2,4), \\
& \frac{\pi}{5}(4,4,4,4,4),
\end{aligned}
$$

$$
\begin{aligned}
& \frac{\pi}{25}(9,16,25,25,25), \\
& \frac{\pi}{91}(36,64,64,100,100), \\
& \frac{\pi}{41}(18,32,32,32,50), \\
& \frac{\pi}{73}(36,64,64,64,64) .
\end{aligned}
$$


Calculation of the function $f$ at each of these points, respectively, shows that its values are, to the nearest hundredth,

Left column: 12.32, 12.27, 12.33, 12.57,

Right column: 12.26, 12.27, 12.33, 12.46 .

These easily exceed the critical value of $6 \cos ^{-1}(-1 / 3) \approx 11.46$ that is the maximum perimeter of any model triple on the unit sphere, so the result follows from Corollary 2.17.

Lemma 4.7. In $\mathbb{R}^{2}$, two bubble triangles with the same signed curvatures of their bounding arcs are isometric to each other.

Proof. This is Lemma 5.7 of [11].

Lemma 4.8. Every bubble triangle $\Delta$ on $\mathbb{S}^{2}$ is isometric to a bubble in some standard triple bubble on $\mathbb{S}^{2}$.

Proof. Stereographically project $\Delta$ to $\Delta^{\prime}$ in $\mathbb{R}^{2}$. (If necessary, rotate $\Delta$ slightly before projecting so that the three curvatures of $\Delta^{\prime}$ are distinct.) Let the largest inward curvature be the reciprocal of one defining radius, and use the other two curvatures as differences to define the other two defining radii of a standard triple bubble in $\mathbb{R}^{2}$. Now project back up to $\mathbb{S}^{2}$.

Proposition 4.9. A competitor $C$ with a lone leaf is not a minimizer of $\mu$ if $\mu(C)<1$.

Proof. Let $\lambda=\mu(C)<1$ and suppose $C$ is a minimizer of $\mu$, nonstandard since $\mu(C)<1$. Suppose $C$ has a lone leaf. A lone leaf of a nonstandard triple bubble must belong to a bubble with an extra component, since the leaf touches only two other components, so if the leaf bubble had no more components, there would be nowhere to place extra components of the other two bubbles, and the whole cluster would have to be standard. Also, the lone leaf and the two components it touches have to be boundary components, so by Corollary 3.3 there cannot be any interior component.

Suppose that we are working in $\mathbb{R}^{2}$. By continuous unification (see Proposition 4.4), if $C$ were a minimizer with $\mu(C)<1$, then a lone leaf in $C$ would have the same signed curvatures as a dilation by a factor of $1 / \lambda$ of the corresponding bubble of $M(C)$. Then by Lemma 4.7, the lone leaf must be isometric to that dilation. But this would give the lone leaf itself (even without adding the other component(s) of the same bubble in $C$ ) more area than the corresponding bubble of $M(C)$, contradicting that (by definition) corresponding areas of bubbles in $C$ and in $M(C)$ must match.

Assume now that we are working in $\mathbb{S}^{2}$.

Case 1: All bubbles and the exterior have area $\pi$. Then all edges are geodesics in both the model $M(C)$ and (by Proposition 4.4) in $C$. Now $C$ has at least one extra component, so the isoperimetric inequality for clusters (Proposition 4.1) gives

$$
P(C) \geq \frac{1}{2}\left(\operatorname{iso}(\pi)+\operatorname{iso}(\pi)+\operatorname{iso}(\pi)+\operatorname{iso}\left(\frac{16}{25} \pi\right)+\operatorname{iso}\left(\frac{9}{25} \pi\right)\right)>12>6 \cos ^{-1}\left(-\frac{1}{3}\right),
$$

so that $C$ is not a minimizer.

Case 2: Some bubble has area less than $\pi$. Then the geodesic curvature $\kappa$ along its exterior is positive, so $\lambda \kappa<\kappa$. Let $K$ be the standard triple bubble whose exterior curvatures are $\lambda$ times those of $C$. Since the exterior curvatures are nonnegative and at least one is positive, the defining radii of $K$ are at least as large, and one strictly larger, than those of $C$, so that by Corollary 2.10 the total area of $K$ is greater than that of $C$.

Now a lone leaf $L$ on $C$ has curvatures matching a bubble $S$ of $K$, so $L$ and $S$ are isometric. The arcs going out from the vertices of $S$ and $L$ have matching curvatures and angles, so if we extend those arcs inward toward the center of $L$, they meet at a point just as they do with $S$. We can thus remove $L$ from $C$ and fill in the bubbles that had been partly covered by $L$, reducing the total area of $C$. Let $C^{\prime}$ be the new cluster. Wichiramala [11] calls this process reduction. 
Since $C$ has no interior component, neither does $C^{\prime}$, so $C^{\prime}$ must also have a lone leaf. If $C^{\prime}$ is not a standard triple bubble, repeat the above process until we have reduced to a standard triple bubble. That triple must, in fact, be $K$. But the area of $C$ is then greater than that of $K$, which is greater than that of $C$, a contradiction.

\section{The 4-daisy}

With no lone leaves, and with a maximum of one interior bubble and two boundary components for each of the other two bubbles, the only remaining nonstandard competitor is a "4-daisy" as depicted in Figure 2.

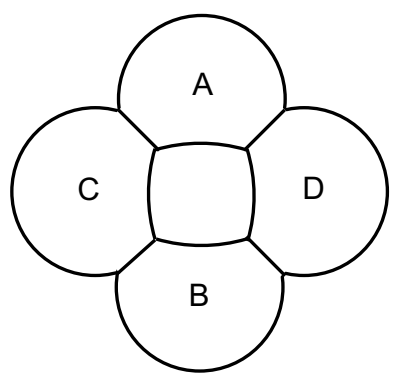

Figure 2

Straightforward calculation establishes the following lemma about relative rates of growth for the perimeter function of a circle on a sphere; the corresponding result in the plane is trivial.

Lemma 5.1. If $u<v$ then the function iso $(t u) /$ iso $(t v)$ is an increasing function of $t$ on $(0,4 \pi / v)$.

Proposition 5.2. A bubble cluster of the type shown in Figure 2 is not a minimizer.

Proof. We present the argument for the sphere; in the plane simply replace the function iso $(t)$ with $\sqrt{4 \pi t}$ and omit the limit argument in the final case.

Let petals $A$ and $B$ belong to bubble 1 , and $C$ and $D$ belong to bubble 2. Let the respective petal areas be $a, b, c$ and $d$. Without loss of generality, $a$ is the largest of these areas, and $c \geq d$. Now by Proposition 2.16, if $b$ is not the smallest area then we can reidentify bubble components $B$ and $D$, bringing the two areas of bubbles 1 and 2 nearer to their average and thus increasing the model perimeter without increasing (and, in fact, decreasing) the competitor perimeter. Thus, we may assume $a \geq c \geq d \geq b$.

Since component $B$ faces the exterior and (components of) two other bubbles, one of these must share at least a third of the perimeter of $B$. If we reidentify $B$ accordingly, the competitor saves at least a third of iso $(b)$, while (by Lemma 3.1) the model saves at most iso $(a+b)-$ iso $(a)$. So, by Corollary 3.3, if $b \leq 9 / 16 a$ then reidentification of $B$ reduces $\mu$. Thus we can assume $b>9 / 16 a$.

Now let $x$ be the total perimeter of the interfaces between petals. We will see that if $x>\operatorname{iso}(a+b)-\operatorname{iso}(2 b)$ then a petal switch reduces $\mu$. Otherwise, a merge of either $A$ or $B$ with center or exterior will reduce $\mu$. Thus, we proceed as follows.

Case 1: $x>$ iso $(a+b)-$ iso( $2 b)$. We need to divide into subcases.

Case 1a: $a+b \geq c+d$. Then we do a switch reidentification between $B$ and $C$. This causes bubble 1 to increase in area and bubble 2 to decrease from $c+d$ to $b+d$. Now reset the bubbles and do a switch reidentification between $B$ and $D$, causing bubble 2 to decrease from area $c+d$ to $b+c$. We will add and compare the results of the savings in order to decide which one of the switches to do. 
Since the interfaces between petals have each been eliminated in one of the switches or the other, the sum of savings for the competitor is at least $x$. The model has saved at most $2 \operatorname{iso}(c+d)-\operatorname{iso}(b+d)-\operatorname{iso}(b+c)$.

Now for a fixed value of $c+d$, by convexity iso $(b+d)+$ iso $(b+c)$ is minimized when $c$ and $d$ are separated the most possible under the hypotheses, namely when $d=b$. So the savings for the model is at most iso $(c+b)-$ iso $(2 b) \leq$ iso $(a+b)-$ iso $(2 b)$. Since $x>$ iso $(a+b)-$ iso $(2 b)$, one of the two switches performed must result in a net decrease in $\mu$.

Case 1b: $c+d \geq a+b$. Then we do a switch reidentification between $A$ and $C$. This causes bubble 2 to increase in area and bubble 1 to decrease from $a+b$ to $c+b$. Reset the bubbles and do a switch between $A$ and $D$. This causes bubble 1 to decrease from $a+b$ to $d+b$. As before, the sum of competitor savings is at least $x$.

For fixed $a$ and $b$, the spread between iso $(a+b)$ and iso $(d+b)$ is largest if $d$ is smallest possible under the hypotheses. So we can assume $c+d=a+b$. As before, convexity shows that the savings for the model is biggest when $c$ and $d$ are furthest apart, making $a=c$ and $b=d$, yielding the same final result as in Case 1 .

Case 2: $x \leq$ iso $(a+b)$ - iso $(2 b)$. Now merge petal $A$ with the center bubble, reset and merge $A$ with the exterior. Do the same for petal $B$. The sum of savings for the competitor is at least iso $(a)+\operatorname{iso}(b)-x \geq \operatorname{iso}(a)+\operatorname{iso}(b)-$ iso $(a+b)+$ iso $(2 b)$, and the sum of savings for the model is at most 4 iso $(a+b)-2$ iso $(a)-2$ iso $(b)$. Thus we need to show that

$$
3 \text { iso }(a)+3 \text { iso }(b)+\operatorname{iso}(2 b)>5 \text { iso }(a+b),
$$

or

$$
3 \frac{\text { iso }(a)}{\text { iso }(a+b)}+3 \frac{\text { iso }(b)}{\text { iso }(a+b)}+\frac{\text { iso }(2 b)}{\text { iso }(a+b)}>5 .
$$

By Lemma 5.1, if we multiply each argument of the iso function by $t$ and let $t$ decrease from 1 toward 0 , the left-hand side is decreasing. The limit as $t$ approaches zero from above is

$$
\frac{3 \sqrt{a}+3 \sqrt{b}+\sqrt{2 b}}{\sqrt{a+b}}=3 \sqrt{1-\eta}+(3+\sqrt{2}) \sqrt{\eta}
$$

where $\eta=b /(a+b)$. For $a \geq b \geq 9 a / 16, \eta \in[9 / 25,1 / 2]$. The second derivative of the above expression by $\eta$ is negative, so its minimum occurs at an endpoint; the respective values at $9 / 25$ and $1 / 2$ are $(21+3 \sqrt{2}) / 5$ and $1+3 \sqrt{2}$, which are both greater than 5 , as desired.

\section{Theorem: The minimizing triple bubbles in $\mathbb{R}^{2}$ and $\mathbb{S}^{2}$}

Theorem 6.1. For every triple of positive real numbers $a_{1}, a_{2}, a_{3}$, with $a_{1}+a_{2}+a_{3}<4 \pi$ in the spherical case, the minimizer of perimeter enclosing those three areas in $\mathbb{R}^{2}$ or $\mathbb{S}^{2}$ is a standard triple bubble.

Proof. First, if we are working in the sphere, switch identities if necessary so that the exterior has area at least as large as each of the main bubbles.

If the theorem is false, then there is some competitor having unified measure $\mu$ less than 1 . Proposition 2.18 guarantees the existence of a unified minimizer $C$; let its unified measure equal $\lambda<1$.

By Corollary 3.3, each interior component of a bubble must have more than 64 percent of the area of that bubble, and each boundary component must have more than 36 percent of the bubble's area. This means that a bubble can only have one interior component and no boundary components, or have one or two boundary components and no interior component.

Clearly not all three bubbles of $C$ could have an interior component without any boundary components. Nor can two bubbles have an interior component, since then there could only exist one boundary component, which would then be a circle enclosing a double bubble. But sliding the double bubble until it touched the circle would give a nonregular configuration, which could then be improved.

If just one bubble has an interior component, the only possibility is for the other two bubbles to each have two boundary components, alternating around the outside. This is a 4-daisy (Figure 2), eliminated by Proposition 5.2. 
Finally, suppose no bubble of $C$ has an interior component. This (together with the connectedness of the exterior, by Proposition 4.6) forces the existence of a lone leaf, in contradiction to Proposition 4.9.

\section{Quadruple bubbles}

It is still an open problem to prove which enclosures of four areas in the plane are perimeter-minimizing. Paolini and Tamaglini [8] have made significant progress on four equal areas. The methods of the present paper look promising as well; these can be sharpened by taking into account a cost that we disregarded here, namely the cost to the model configuration of inflating bubbles that have been reidentified. Computer assistance appears to be of value in this direction.

The proposed minimizer places the two bubbles of largest area together and locates the two smallest bubbles on opposite sides of the large ones. One complication with unification for quadruple bubbles is the discontinuity in the shape of proposed minimizers that occurs where the second and third areas, in order of size, are equal; if the third area then grows larger than the second, the second and third bubbles switch places. This changes their curvatures slightly, causing the derivatives in length to only exist as one-sided derivatives at such a point and weakening Proposition 4.4.

\section{References}

[1] Amilibia, A. Montesinos. Existence and uniqueness of standard bubble clusters of given volumes in $\mathbf{R}^{N}$. Asian Journal of Mathematics 5, no. 1 (January 1, 2001): 25.

[2] Bleicher, M. N., Isoperimetric networks in the Euclidean plane, Studia Scientiarum Mathematicarum Hungarica 31 (1996) 455-478.

[3] C. Cox, L. Harrison, M. Hutchings, S. Kim, J. Light, A. Mauer, M. Tilton, The shortest enclosure of three connected areas in $\mathbb{R}^{2}$, Real Anal. Exchange 20 (1994/95), 313-335.

[4] Foisy, Joel; Alfaro, Manuel; Brock, Jeffrey; Hodges, Nickelous; Zimba, Jason: The standard double soap bubble in $\mathbf{R}^{2}$ uniquely minimizes perimeter. Pacific J. Math., 159, no. 1, 47-59.

[5] Lawlor, Gary R. Double bubbles for immiscible fluids in $\mathbb{R}^{n}$. J. Geom. Anal. 24 (2014), no. 1, 190-204.

[6] Masters, Joseph D. The perimeter-miniming enclosure of two areas in $\mathbb{S}^{2}$, Real Anal. Exch. 22 (1996-97) no. 2, 645-654.

[7] Morgan, Frank: Soap bubbles in $\mathbb{R}^{2}$ and on surfaces, Pacific J. Math., 165 (1994), 347-361.

[8] Paolini, Emanuele, Tamagnini, Andrea. Minimal clusters of four planar regions with the same area. ESAIM: Control, Optimisation and Calculus of Variations, 2018010124 (no. 3):1303-1331.

[9] Rubinstein, J. H., Thomas, D. A.: Graham's problem on shortest networks for points on a circle. Algorithmica 7, 193-218 (1992)

[10] Tamagnini, A.: Planar Clusters. Ph.D. Thesis, Universita degli Studi di Firenze (2015).

[11] Wichiramala, Wacharin: Proof of the planar triple bubble conjecture. J. Reine Angew. Math. 567 (2004), 1-49. 